\title{
Several Integrability Formulas of Special Functions. Part II
}

\author{
Bo Li \\ Qingdao University of Science \\ and Technology \\ China \\ Yanhong Men \\ Qingdao University of Science \\ and Technology \\ China
}

\author{
Yanping Zhuang \\ Qingdao University of Science \\ and Technology \\ China \\ Xiquan Liang \\ Qingdao University of Science \\ and Technology \\ China
}

Summary. In this article, we give several differentiation and integrability formulas of special and composite functions including the trigonometric function, the hyperbolic function and the polynomial function [3].

MML identifier: INTEGR11, version: $\underline{7.11 .01 \quad 4.117 .1046}$

The articles [10], [23], [19], [21], [22], [1], [8], [15], [9], [2], [4], [17], [5], [13], [16], [14], [18], [7], [12], [20], [6], and [11] provide the terminology and notation for this paper.

\section{Differentiation Formulas}

For simplicity, we adopt the following rules: $r, x, a, b$ denote real numbers, $n$, $m$ denote elements of $\mathbb{N}, A$ denotes a closed-interval subset of $\mathbb{R}$, and $Z$ denotes an open subset of $\mathbb{R}$.

One can prove the following propositions:

(1)(i) $\quad\left(\frac{1}{2} \square+0\right)-\frac{1}{4}$ ((the function sin) $\left.\cdot(2 \square+0)\right)$ is differentiable on $\mathbb{R}$, and

(ii) for every $x$ holds $\left(\left(\frac{1}{2} \square+0\right)-\frac{1}{4}((\text { the function } \sin ) \cdot(2 \square+0))\right)^{\prime}(x)=$ $(\sin x)^{2}$. 
(2)(i) $\quad\left(\frac{1}{2} \square+0\right)+\frac{1}{4}$ ((the function $\left.\left.\sin \right) \cdot(2 \square+0)\right)$ is differentiable on $\mathbb{R}$, and

(ii) for every $x$ holds $\left(\left(\frac{1}{2} \square+0\right)+\frac{1}{4}\right.$ ((the function $\left.\left.\left.\sin \right) \cdot(2 \square+0)\right)\right)_{\uparrow \mathbb{R}}^{\prime}(x)=$ $(\cos x)^{2}$.

(3) $\frac{1}{n+1}\left(\left(\square^{n+1}\right) \cdot(\right.$ the function $\left.\sin )\right)$ is differentiable on $\mathbb{R}$ and for every $x$ holds $\left.\left(\frac{1}{n+1} \text { (the function } \sin \right)^{n+1}\right)_{\mathbb{R}}^{\prime}(x)=(\sin x)^{n} \cdot \cos x$.

(4)(i) $\quad\left(-\frac{1}{n+1}\right)\left(\left(\square^{n+1}\right) \cdot(\right.$ the function cos $\left.)\right)$ is differentiable on $\mathbb{R}$, and

(ii) for every $x$ holds $\left.\left(\left(-\frac{1}{n+1}\right) \text { (the function } \cos \right)^{n+1}\right)_{\mathbb{R}}^{\prime}(x)=(\cos x)^{n} \cdot \sin x$.

(5) Suppose $m+n \neq 0$ and $m-n \neq 0$. Then

(i) $\frac{1}{2 \cdot(m+n)}(($ the function $\sin ) \cdot((m+n) \square+0))+\frac{1}{2 \cdot(m-n)}$ ((the function sin) $\cdot((m-n) \square+0))$ is differentiable on $\mathbb{R}$, and

(ii) for every $x$ holds $\left(\frac{1}{2 \cdot(m+n)}((\right.$ the function $\sin ) \cdot((m+n) \square+0))+$ $\frac{1}{2 \cdot(m-n)}(($ the function $\left.\sin ) \cdot((m-n) \square+0))\right)_{\uparrow \mathbb{R}}^{\prime}(x)=\cos (m \cdot x) \cdot \cos (n \cdot x)$.

(6) Suppose $m+n \neq 0$ and $m-n \neq 0$. Then

(i) $\frac{1}{2 \cdot(m-n)}(($ the function $\sin ) \cdot((m-n) \square+0))-\frac{1}{2 \cdot(m+n)}(($ the function sin $)$ $\cdot((m+n) \square+0))$ is differentiable on $\mathbb{R}$, and

(ii) for every $x$ holds $\left(\frac{1}{2 \cdot(m-n)}((\right.$ the function $\sin ) \cdot((m-n) \square+0))-$ $\frac{1}{2 \cdot(m+n)}(($ the function sin $\left.) \cdot((m+n) \square+0))\right)_{\mathbb{R} \mathbb{R}}^{\prime}(x)=\sin (m \cdot x) \cdot \sin (n \cdot x)$.

(7) Suppose $m+n \neq 0$ and $m-n \neq 0$. Then

(i) $\quad-\frac{1}{2 \cdot(m+n)}(($ the function $\cos ) \cdot((m+n) \square+0))-\frac{1}{2 \cdot(m-n)}(($ the function $\cos ) \cdot((m-n) \square+0))$ is differentiable on $\mathbb{R}$, and

(ii) for every $x$ holds $\left(-\frac{1}{2 \cdot(m+n)}((\right.$ the function $\cos ) \cdot((m+n) \square+0))-$ $\frac{1}{2 \cdot(m-n)}(($ the function $\left.\cos ) \cdot((m-n) \square+0))\right)_{\uparrow \mathbb{R}}^{\prime}(x)=\sin (m \cdot x) \cdot \cos (n \cdot x)$.

(8) Suppose $n \neq 0$. Then

(i) $\frac{1}{n^{2}}(($ the function $\sin ) \cdot(n \square+0))-\left(\frac{1}{n} \square+0\right)(($ the function $\cos ) \cdot(n \square+0))$ is differentiable on $\mathbb{R}$, and

(ii) for every $x$ holds $\left(\frac{1}{n^{2}}((\right.$ the function $\sin ) \cdot(n \square+0))-\left(\frac{1}{n} \square+0\right)$ ((the function $\cos ) \cdot(n \square+0)))_{\uparrow \mathbb{R}}^{\prime}(x)=x \cdot \sin (n \cdot x)$.

(9) Suppose $n \neq 0$. Then

(i) $\frac{1}{n^{2}}(($ the function $\cos ) \cdot(n \square+0))+\left(\frac{1}{n} \square+0\right)(($ the function sin $) \cdot(n \square+0))$ is differentiable on $\mathbb{R}$, and

(ii) for every $x$ holds $\left(\frac{1}{n^{2}}((\right.$ the function cos $) \cdot(n \square+0))+\left(\frac{1}{n} \square+0\right)(($ the function sin) $\cdot(n \square+0)))_{\mathbb{R}}^{\prime}(x)=x \cdot \cos (n \cdot x)$.

(10)(i) $\quad(1 \square+0)$ (the function cosh) - the function sinh is differentiable on $\mathbb{R}$, and

(ii) for every $x$ holds $((1 \square+0)$ (the function cosh)-the function $\sinh )^{\prime}(x)=x \cdot \sinh x$.

(11)(i) $\quad(1 \square+0)$ (the function sinh)-the function cosh is differentiable on $\mathbb{R}$, and 
(ii) for every $x$ holds $((1 \square+0)$ (the function sinh)-the function $\cosh )^{\prime}(x)=x \cdot \cosh x$.

(12) If $a \cdot(n+1) \neq 0$, then $\frac{1}{a \cdot(n+1)}(a \square+b)^{n+1}$ is differentiable on $\mathbb{R}$ and for every $x$ holds $\left(\frac{1}{a \cdot(n+1)}(a \square+b)^{n+1}\right)_{\mathbb{R}}^{\prime}(x)=(a \cdot x+b)^{n}$.

\section{INTEGRABILITY FormulaS}

Next we state a number of propositions:

(13) $\int_{A}(\text { the function } \sin )^{2}(x) d x=\frac{1}{2} \cdot \sup A-\frac{1}{4} \cdot \sin (2 \cdot \sup A)-\left(\frac{1}{2} \cdot \inf A-\right.$ $\left.\frac{1}{4} \cdot \sin (2 \cdot \inf A)\right)$.

(14) $\int_{[0, \pi]}(\text { the function } \sin )^{2}(x) d x=\frac{\pi}{2}$.

(15) $\int_{[0,2 \cdot \pi]}(\text { the function } \sin )^{2}(x) d x=\pi$.

(16) $\int_{A}(\text { the function } \cos )^{2}(x) d x=\left(\frac{1}{2} \cdot \sup A+\frac{1}{4} \cdot \sin (2 \cdot \sup A)\right)-\left(\frac{1}{2} \cdot \inf A+\right.$ $\left.\frac{1}{4} \cdot \sin (2 \cdot \inf A)\right)$.

(17) $\int_{[0, \pi]}(\text { the function } \cos )^{2}(x) d x=\frac{\pi}{2}$.

(18) $\int_{[0,2 \cdot \pi]}(\text { the function } \cos )^{2}(x) d x=\pi$.

(19) $\int_{A}\left((\text { the function } \sin )^{n}(\right.$ the function $\left.\cos )\right)(x) d x=\frac{1}{n+1} \cdot(\sin \sup A)^{n+1}-$ $\frac{A}{n+1} \cdot(\sin \inf A)^{n+1}$.

(20) $\int_{[0, \pi]}\left((\text { the function sin })^{n}\right.$ (the function $\left.\left.\cos \right)\right)(x) d x=0$.

(21) $\int_{[0,2 \cdot \pi]}\left((\text { the function } \sin )^{n}\right.$ (the function $\left.\left.\cos \right)\right)(x) d x=0$.

(22) $\int_{A}\left((\text { the function } \cos )^{n}(\right.$ the function $\left.\sin )\right)(x) d x=\left(-\frac{1}{n+1}\right) \cdot(\cos \sup A)^{n+1}-$ $\left(-\frac{1}{n+1}\right) \cdot(\cos \inf A)^{n+1}$. 
(23) $\int_{[0,2 \cdot \pi]}\left((\text { the function } \cos )^{n}(\right.$ the function $\left.\sin )\right)(x) d x=0$.

(24) $\int_{\left[-\frac{\pi}{2}, \frac{\pi}{2}\right]}\left((\text { the function } \cos )^{n}\right.$ (the function $\left.\left.\sin \right)\right)(x) d x=0$.

(25) Suppose $m+n \neq 0$ and $m-n \neq 0$. Then $\int_{A}((($ the function cos $) \cdot(m \square+0))(($ the function $\cos ) \cdot(n \square+0)))(x) d x=$ $\left(\frac{1}{2 \cdot(m+n)} \cdot \sin ((m+n) \cdot \sup A)+\frac{1}{2 \cdot(m-n)} \cdot \sin ((m-n) \cdot \sup A)\right)-$ $\left(\frac{1}{2 \cdot(m+n)} \cdot \sin ((m+n) \cdot \inf A)+\frac{1}{2 \cdot(m-n)} \cdot \sin ((m-n) \cdot \inf A)\right)$.

(26) Suppose $m+n \neq 0$ and $m-n \neq 0$. Then $\int_{A}((($ the function $\sin ) \cdot(m \square+0))(($ the function $\sin ) \cdot(n \square+0)))(x) d x=$ $\frac{1}{2 \cdot(m-n)} \cdot \sin ((m-n) \cdot \sup A)-\frac{1}{2 \cdot(m+n)} \cdot \sin ((m+n) \cdot \sup A)-$ $\left(\frac{1}{2 \cdot(m-n)} \cdot \sin ((m-n) \cdot \inf A)-\frac{1}{2 \cdot(m+n)} \cdot \sin ((m+n) \cdot \inf A)\right)$.

(27) Suppose $m+n \neq 0$ and $m-n \neq 0$. Then $\int_{A}((($ the function $\sin ) \cdot(m \square+0))(($ the function $\cos ) \cdot(n \square+0)))(x) d x=$ $-\frac{1}{2 \cdot(m+n)} \cdot \cos ((m+n) \cdot \sup A)-\frac{1}{2 \cdot(m-n)} \cdot \cos ((m-n) \cdot \sup A)-$ $\left(-\frac{1}{2 \cdot(m+n)} \cdot \cos ((m+n) \cdot \inf A)-\frac{1}{2 \cdot(m-n)} \cdot \cos ((m-n) \cdot \inf A)\right)$.

(28) If $n \neq 0$, then $\int_{A}((1 \square+0)(($ the function $\sin ) \cdot(n \square+0)))(x) d x=\frac{1}{n^{2}}$. $\sin (n \cdot \sup A)-\frac{1}{n} \cdot \sup A \cdot \cos (n \cdot \sup A)-\left(\frac{1}{n^{2}} \cdot \sin (n \cdot \inf A)-\frac{1}{n} \cdot \inf A\right.$. $\cos (n \cdot \inf A))$.

(29) If $n \neq 0$, then $\int_{A}((1 \square+0)(($ the function $\cos ) \cdot(n \square+0)))(x) d x=\left(\frac{1}{n^{2}}\right.$. $\left.\cos (n \cdot \sup A)+\frac{1}{n} \cdot \sup A \cdot \sin (n \cdot \sup A)\right)-\left(\frac{1}{n^{2}} \cdot \cos (n \cdot \inf A)+\frac{1}{n} \cdot \inf A \cdot\right.$ $\sin (n \cdot \inf A))$.

(30) $\int_{A}((1 \square+0)($ the function $\sinh ))(x) d x=\sup A \cdot \cosh \sup A-\sinh \sup A-$ (inf $A \cdot \cosh \inf A-\sinh \inf A$ ).

(31) $\int_{A}((1 \square+0)($ the function $\cosh ))(x) d x=\sup A \cdot \sinh \sup A-\cosh \sup A-$ $(\inf A \cdot \sinh \inf A-\cosh \inf A)$. 
(32) If $a \cdot(n+1) \neq 0$, then $\int_{A}(a \square+b)^{n}(x) d x=\frac{1}{a \cdot(n+1)} \cdot(a \cdot \sup A+b)^{n+1}-$ $\frac{1}{a \cdot(n+1)} \cdot(a \cdot \inf A+b)^{n+1}$.

\section{ADDENDA}

In the sequel $f, f_{1}, f_{2}, f_{3}, g$ are partial functions from $\mathbb{R}$ to $\mathbb{R}$.

The following propositions are true:

(33) If $Z \subseteq \operatorname{dom}\left(\frac{1}{2} f\right)$ and $f=\square^{2}$, then $\frac{1}{2} f$ is differentiable on $Z$ and for every $x$ such that $x \in Z$ holds $\left(\frac{1}{2} f\right)^{\prime}{ }_{Z}(x)=x$.

(34) If $A \subseteq Z=\operatorname{dom}\left(\frac{1}{2}\left(\square^{2}\right)\right)$, then $\int_{A} \operatorname{id}_{Z}(x) d x=\frac{1}{2} \cdot(\sup A)^{2}-\frac{1}{2} \cdot(\inf A)^{2}$.

(35) Suppose $A \subseteq Z$ and for every $x$ such that $x \in Z$ holds $g(x)=x$ and $g(x) \neq 0$ and $f(x)=-\frac{1}{x^{2}}$ and $Z=\operatorname{dom} g$ and $\operatorname{dom} f=Z$ and $f \nmid A$ is continuous. Then $\int_{A} f(x) d x=(\sup A)^{-1}-(\inf A)^{-1}$.

(36) Suppose that

(i) $A \subseteq Z$,

(ii) $f_{1}=\square^{2}$,

(iii) for every $x$ such that $x \in Z$ holds $f_{2}(x)=1$ and $x \neq 0$ and $f(x)=$ $\frac{2 \cdot x}{\left(1+x^{2}\right)^{2}}$

(iv) $\operatorname{dom}\left(\frac{f_{1}}{f_{2}+f_{1}}\right)=Z$,

(v) $Z=\operatorname{dom} f$, and

(vi) $f \uparrow A$ is continuous.

Then $\int_{A} f(x) d x=\left(\frac{f_{1}}{f_{2}+f_{1}}\right)(\sup A)-\left(\frac{f_{1}}{f_{2}+f_{1}}\right)(\inf A)$.

(37) Suppose $Z \subseteq \operatorname{dom}(($ the function $\tan )+($ the function sec) $)$ and for every $x$ such that $x \in Z$ holds $1+\sin x \neq 0$ and $1-\sin x \neq 0$. Then

(i) (the function tan) + (the function sec) is differentiable on $Z$, and

(ii) for every $x$ such that $x \in Z$ holds ((the function tan)+(the function sec) $)_{\uparrow Z}^{\prime}(x)=\frac{1}{1-\sin x}$.

(38) Suppose that

(i) $A \subseteq Z$,

(ii) for every $x$ such that $x \in Z$ holds $1+\sin x \neq 0$ and $1-\sin x \neq 0$ and $f(x)=\frac{1}{1-\sin x}$,

(iii) $\operatorname{dom}(($ the function $\tan )+($ the function $\mathrm{sec}))=Z$,

(iv) $Z=\operatorname{dom} f$, and

(v) $f \uparrow A$ is continuous. 
Then $\int_{A} f(x) d x=(\tan \sup A+\sec \sup A)-(\tan \inf A+\sec \inf A)$.

(39) Suppose $Z \subseteq \operatorname{dom}(($ the function tan $)-($ the function sec) $)$ and for every $x$ such that $x \in Z$ holds $1+\sin x \neq 0$ and $1-\sin x \neq 0$. Then

(i) (the function tan)-(the function sec) is differentiable on $Z$, and

(ii) for every $x$ such that $x \in Z$ holds ((the function tan)-(the function sec) $)_{\uparrow Z}^{\prime}(x)=\frac{1}{1+\sin x}$.

(40) Suppose that

(i) $A \subseteq Z$,

(ii) for every $x$ such that $x \in Z$ holds $1+\sin x \neq 0$ and $1-\sin x \neq 0$ and $f(x)=\frac{1}{1+\sin x}$,

(iii) $\operatorname{dom}(($ the function $\tan )-($ the function $\sec ))=Z$,

(iv) $Z=\operatorname{dom} f$, and

(v) $f \uparrow A$ is continuous.

Then $\int_{A} f(x) d x=\tan \sup A-\sec \sup A-(\tan \inf A-\sec \inf A)$.

(41) Suppose $Z \subseteq \operatorname{dom}$ (-the function cot + the function cosec) and for every $x$ such that $x \in Z$ holds $1+\cos x \neq 0$ and $1-\cos x \neq 0$. Then

(i) -the function cot + the function cosec is differentiable on $Z$, and

(ii) for every $x$ such that $x \in Z$ holds (-the function cot + the function $\operatorname{cosec})^{\prime}{ }_{Z}(x)=\frac{1}{1+\cos x}$.

(42) Suppose that

(i) $A \subseteq Z$,

(ii) for every $x$ such that $x \in Z$ holds $1+\cos x \neq 0$ and $1-\cos x \neq 0$ and $f(x)=\frac{1}{1+\cos x}$,

(iii) $\operatorname{dom}(-$ the function $\cot +$ the function $\operatorname{cosec})=Z$,

(iv) $Z=\operatorname{dom} f$, and

(v) $f \uparrow A$ is continuous.

Then $\int_{A} f(x) d x=(-\cot \sup A+\operatorname{cosec} \sup A)-(-\cot \inf A+\operatorname{cosec} \inf A)$.

(43) Suppose $Z \subseteq \operatorname{dom}$ (-the function cot - the function cosec) and for every $x$ such that $x \in Z$ holds $1+\cos x \neq 0$ and $1-\cos x \neq 0$. Then

(i) -the function cot - the function cosec is differentiable on $Z$, and

(ii) for every $x$ such that $x \in Z$ holds (-the function cot - the function $\operatorname{cosec})^{\prime}{ }_{Z}(x)=\frac{1}{1-\cos x}$.

(44) Suppose that

(i) $A \subseteq Z$,

(ii) for every $x$ such that $x \in Z$ holds $1+\cos x \neq 0$ and $1-\cos x \neq 0$ and $f(x)=\frac{1}{1-\cos x}$,

(iii) $\operatorname{dom}(-$ the function $\cot -$ the function $\operatorname{cosec})=Z$,

(iv) $Z=\operatorname{dom} f$, and 
(v) $f \uparrow A$ is continuous.

Then $\int_{A} f(x) d x=-\cot \sup A-\operatorname{cosec} \sup A-(-\cot \inf A-\operatorname{cosec} \inf A)$.

(45) Suppose that

(i) $A \subseteq Z$,

(ii) $Z \subseteq]-1,1[$,

(iii) for every $x$ such that $x \in Z$ holds $f(x)=\frac{1}{1+x^{2}}$,

(iv) $\operatorname{dom}($ the function $\arctan )=Z$,

(v) $Z=\operatorname{dom} f$, and

(vi) $f \nmid A$ is continuous.

Then $\int_{A} f(x) d x=\arctan \sup A-\arctan \inf A$.

(46) Suppose that

(i) $A \subseteq Z$,

(ii) $Z \subseteq]-1,1[$,

(iii) for every $x$ such that $x \in Z$ holds $f(x)=\frac{r}{1+x^{2}}$,

(iv) $\operatorname{dom}(r$ the function $\arctan )=Z$,

(v) $Z=\operatorname{dom} f$, and

(vi) $f \uparrow A$ is continuous.

Then $\int_{A} f(x) d x=r \cdot \arctan \sup A-r \cdot \arctan \inf A$.

(47) Suppose that

(i) $A \subseteq Z$,

(ii) $Z \subseteq]-1,1[$,

(iii) for every $x$ such that $x \in Z$ holds $f(x)=-\frac{1}{1+x^{2}}$,

(iv) $\operatorname{dom}($ the function arccot) $=Z$,

(v) $Z=\operatorname{dom} f$, and

(vi) $f \uparrow A$ is continuous.

Then $\int_{A} f(x) d x=\operatorname{arccot} \sup A-\operatorname{arccot} \inf A$.

(48) Suppose that

(i) $A \subseteq Z$,

(ii) $Z \subseteq]-1,1[$,

(iii) for every $x$ such that $x \in Z$ holds $f(x)=-\frac{r}{1+x^{2}}$,

(iv) $\operatorname{dom}(r$ the function $\operatorname{arccot})=Z$,

(v) $Z=\operatorname{dom} f$, and

(vi) $f \uparrow A$ is continuous.

Then $\int_{A} f(x) d x=r \cdot \operatorname{arccot} \sup A-r \cdot \operatorname{arccot} \inf A$.

(49) Suppose $Z \subseteq \operatorname{dom}\left(\left(\operatorname{id}_{Z}+\right.\right.$ the function cot $)$-the function cosec $)$ and for every $x$ such that $x \in Z$ holds $1+\cos x \neq 0$ and $1-\cos x \neq 0$. Then 
(i) (id $Z+$ the function cot) - the function cosec is differentiable on $Z$, and

(ii) for every $x$ such that $x \in Z$ holds ((id $Z$ the function cot)-the function $\operatorname{cosec})^{\prime}{ }_{Z}(x)=\frac{\cos x}{1+\cos x}$.

(50) Suppose that

(i) $A \subseteq Z$,

(ii) for every $x$ such that $x \in Z$ holds $1+\cos x \neq 0$ and $1-\cos x \neq 0$ and $f(x)=\frac{\cos x}{1+\cos x}$,

(iii) $\operatorname{dom}\left(\left(\operatorname{id}_{Z}+\right.\right.$ the function cot $)-$ the function cosec $)=Z$,

(iv) $Z=\operatorname{dom} f$, and

(v) $f \uparrow A$ is continuous.

Then $\int_{A} f(x) d x=(\sup A+\cot \sup A)-\operatorname{cosec} \sup A-((\inf A+\cot \inf A)-$ $\operatorname{cosec} \inf A$ ).

(51) Suppose $Z \subseteq \operatorname{dom}\left(\operatorname{id}_{Z}+\right.$ the function cot+the function cosec) and for every $x$ such that $x \in Z$ holds $1+\cos x \neq 0$ and $1-\cos x \neq 0$. Then

(i) $\operatorname{id}_{Z}+$ the function cot+the function cosec is differentiable on $Z$, and

(ii) for every $x$ such that $x \in Z$ holds $\left(\operatorname{id}_{Z}+\right.$ the function $\cot +$ the function $\operatorname{cosec})_{{ }^{\prime}}^{\prime}(x)=\frac{\cos x}{\cos x-1}$.

(52) Suppose that

(i) $A \subseteq Z$,

(ii) for every $x$ such that $x \in Z$ holds $1+\cos x \neq 0$ and $1-\cos x \neq 0$ and $f(x)=\frac{\cos x}{\cos x-1}$,

(iii) $\operatorname{dom}\left(\mathrm{id}_{Z}+\right.$ the function $\cot +$ the function $\left.\operatorname{cosec}\right)=Z$,

(iv) $Z=\operatorname{dom} f$, and

(v) $f \uparrow A$ is continuous.

Then $\int_{A} f(x) d x=(\sup A+\cot \sup A+\operatorname{cosec} \sup A)-(\inf A+\cot \inf A+$ $\operatorname{cosec} \inf A)$.

(53) Suppose $Z \subseteq \operatorname{dom}\left(\left(\operatorname{id}_{Z}\right.\right.$ - the function $\left.\tan \right)+$ the function sec $)$ and for every $x$ such that $x \in Z$ holds $1+\sin x \neq 0$ and $1-\sin x \neq 0$. Then

(i) $\left(\operatorname{id}_{Z}\right.$ - the function $\left.\tan \right)+$ the function sec is differentiable on $Z$, and

(ii) for every $x$ such that $x \in Z$ holds ((id $Z$-the function $\tan )+$ the function sec $)_{\lceil Z}^{\prime}(x)=\frac{\sin x}{\sin x+1}$

(54) Suppose that

(i) $A \subseteq Z$,

(ii) for every $x$ such that $x \in Z$ holds $1+\sin x \neq 0$ and $1-\sin x \neq 0$ and $f(x)=\frac{\sin x}{1+\sin x}$,

(iii) $Z \subseteq \operatorname{dom}\left(\left(\mathrm{id}_{Z}-\right.\right.$ the function $\left.\tan \right)+$ the function sec $)$,

(iv) $Z=\operatorname{dom} f$, and

(v) $f \uparrow A$ is continuous. 
Then $\int_{A} f(x) d x=((\sup A-\tan \sup A)+\sec \sup A)-((\inf A-\tan \inf A)+$ $\sec \inf A)$.

(55) Suppose $Z \subseteq \operatorname{dom}\left(\mathrm{id}_{Z}\right.$ - the function tan-the function sec) and for every $x$ such that $x \in Z$ holds $1+\sin x \neq 0$ and $1-\sin x \neq 0$. Then

(i) $\quad \mathrm{id}_{Z}$ - the function tan-the function sec is differentiable on $Z$, and

(ii) for every $x$ such that $x \in Z$ holds (id $Z$ - the function tan-the function sec $)^{\prime}(x)=\frac{\sin x}{\sin x-1}$.

(56) Suppose that

(i) $A \subseteq Z$,

(ii) for every $x$ such that $x \in Z$ holds $1+\sin x \neq 0$ and $1-\sin x \neq 0$ and $f(x)=\frac{\sin x}{\sin x-1}$,

(iii) $Z \subseteq \operatorname{dom}\left(\operatorname{id}_{Z}-\right.$ the function $\tan -$ the function sec),

(iv) $Z=\operatorname{dom} f$, and

(v) $f\lceil A$ is continuous.

Then $\int_{A} f(x) d x=\sup A-\tan \sup A-\sec \sup A-(\inf A-\tan \inf A-$ $\sec \inf A)$.

(57) Suppose $Z \subseteq \operatorname{dom}\left((\right.$ the function $\left.\tan )-\mathrm{id}_{Z}\right)$. Then (the function $\tan )-\mathrm{id}_{Z}$ is differentiable on $Z$ and for every $x$ such that $x \in Z$ holds $\left((\text { the function } \tan )-\mathrm{id}_{Z}\right)_{\uparrow Z}^{\prime}(x)=(\tan x)^{2}$.

(58) Suppose that

(i) $A \subseteq Z$,

(ii) for every $x$ such that $x \in Z$ holds (the function $\cos )(x)>0$ and $f(x)=(\tan x)^{2}$,

(iii) $Z \subseteq \operatorname{dom}\left((\right.$ the function $\left.\tan )-\mathrm{id}_{Z}\right)$,

(iv) $Z=\operatorname{dom} f$, and

(v) $f \uparrow A$ is continuous.

Then $\int_{A} f(x) d x=\tan \sup A-\sup A-(\tan i n f A-\inf A)$.

(59) Suppose $Z \subseteq \operatorname{dom}\left(-\right.$ the function $\left.\cot -\mathrm{id}_{Z}\right)$. Then - the function cot $\operatorname{id}_{Z}$ is differentiable on $Z$ and for every $x$ such that $x \in Z$ holds $\left(\text { - the function } \cot -\mathrm{id}_{Z}\right)_{\uparrow Z}^{\prime}(x)=(\cot x)^{2}$.

(60) Suppose that

(i) $A \subseteq Z$,

(ii) for every $x$ such that $x \in Z$ holds (the function $\sin )(x)>0$ and $f(x)=(\cot x)^{2}$,

(iii) $Z \subseteq \operatorname{dom}\left(-\right.$ the function $\left.\cot -\mathrm{id}_{Z}\right)$,

(iv) $Z=\operatorname{dom} f$, and

(v) $f\lceil A$ is continuous. 
Then $\int_{A} f(x) d x=-\cot \sup A-\sup A-(-\cot \inf A-\inf A)$.

(61) Suppose $A \subseteq Z$ and for every $x$ such that $x \in Z$ holds $f(x)=\frac{1}{(\cos x)^{2}}$ and $\cos x \neq 0$ and $\operatorname{dom}$ (the function $\tan )=Z=\operatorname{dom} f$ and $f \uparrow A$ is continuous. Then $\int_{A} f(x) d x=\tan \sup A-\tan \inf A$.

(62) Suppose $A \subseteq Z$ and for every $x$ such that $x \in Z$ holds $f(x)=-\frac{1}{(\sin x)^{2}}$ and $\sin x \neq 0$ and $\operatorname{dom}$ (the function cot) $=Z=\operatorname{dom} f$ and $f\lceil A$ is continuous. Then $\int_{A} f(x) d x=\cot \sup A-\cot \inf A$.

(63) Suppose $A \subseteq Z$ and for every $x$ such that $x \in Z$ holds $f(x)=\frac{\sin x-(\cos x)^{2}}{(\cos x)^{2}}$ and $Z \subseteq \operatorname{dom}$ ((the function sec)-id $Z$ ) and $Z=\operatorname{dom} f$ and $f\lceil A$ is continuous. Then $\int_{A} f(x) d x=\sec \sup A-\sup A-(\operatorname{secinf} A-\inf A)$.

(64) Suppose that

(i) $A \subseteq Z$,

(ii) for every $x$ such that $x \in Z$ holds $f(x)=\frac{\cos x-(\sin x)^{2}}{(\sin x)^{2}}$,

(iii) $Z \subseteq \operatorname{dom}\left(-\right.$ the function $\operatorname{cosec}-\mathrm{id}_{Z}$ ),

(iv) $Z=\operatorname{dom} f$, and

(v) $f \uparrow A$ is continuous.

Then $\int_{A} f(x) d x=-\operatorname{cosec} \sup A-\sup A-(-\operatorname{cosec} \inf A-\inf A)$.

The following propositions are true:

(65) Suppose that

(i) $A \subseteq Z$,

(ii) for every $x$ such that $x \in Z$ holds $\sin x>0$,

(iii) $Z \subseteq \operatorname{dom}(($ the function $\ln ) \cdot($ the function sin $)$ ),

(iv) $Z=\operatorname{dom}$ (the function cot), and

(v) (the function $\cot )\lceil A$ is continuous.

Then $\int_{A}($ the function cot $)(x) d x=\ln \sin \sup A-\ln \sin \inf A$.

(66) Suppose that

(i) $A \subseteq Z$,

(ii) $Z \subseteq]-1,1[$,

(iii) for every $x$ such that $x \in Z$ holds $f(x)=\frac{\arcsin x}{\sqrt{1-x^{2}}}$,

(iv) $\left.Z \subseteq \operatorname{dom}\left(\frac{1}{2} \text { (the function } \arcsin \right)^{2}\right)$,

(v) $Z=\operatorname{dom} f$, and

(vi) $f \uparrow A$ is continuous. 
Then $\int_{A} f(x) d x=\frac{1}{2} \cdot(\arcsin \sup A)^{2}-\frac{1}{2} \cdot(\arcsin \inf A)^{2}$.

(67) Suppose that

(i) $A \subseteq Z$,

(ii) $Z \subseteq]-1,1[$,

(iii) for every $x$ such that $x \in Z$ holds $f(x)=-\frac{\arccos x}{\sqrt{1-x^{2}}}$,

(iv) $Z \subseteq \operatorname{dom}\left(\frac{1}{2} \text { (the function } \arccos \right)^{2}$ ),

(v) $Z=\operatorname{dom} f$, and

(vi) $f \uparrow A$ is continuous.

Then $\int_{A} f(x) d x=\frac{1}{2} \cdot(\arccos \sup A)^{2}-\frac{1}{2} \cdot(\operatorname{arccosinf} A)^{2}$.

(68) $A \subseteq Z \subseteq]-1,1\left[\right.$ and $f=f_{1}-f_{2}$ and $f_{2}=\square^{2}$ and for every $x$ such that $x \in Z$ holds $f_{1}(x)=1$ and $f(x)>0$ and $x \neq 0$ and dom (the function $\arcsin )=Z \subseteq \operatorname{dom}\left(\operatorname{id}_{Z}(\right.$ the function $\left.\arcsin )+f^{\frac{1}{2}}\right)$.

(69) Suppose that $A \subseteq Z \subseteq]-1,1\left[\right.$ and $f=f_{1}-f_{2}$ and $f_{2}=\square^{2}$ and for every $x$ such that $x \in Z$ holds $f_{1}(x)=a^{2}$ and $f(x)>0$ and $f_{3}(x)=\frac{x}{a}$ and $-1<f_{3}(x)<1$ and $x \neq 0$ and $a>0$ and $\operatorname{dom}\left((\right.$ the function $\left.\arcsin ) \cdot f_{3}\right)=$ $Z \subseteq \operatorname{dom}\left(\operatorname{id}_{Z}\left((\right.\right.$ the function $\left.\left.\arcsin ) \cdot f_{3}\right)+\left(\square^{\frac{1}{2}}\right) \cdot f\right)$ and ((the function $\left.\arcsin ) \cdot f_{3}\right)\left\lceil A\right.$ is continuous. Then $\int_{A}\left((\right.$ the function $\left.\arcsin ) \cdot f_{3}\right)(x) d x=$ $\left(\sup A \cdot \arcsin \left(\frac{\sup A}{a}\right)+f(\sup A)^{\frac{1}{2}}\right)-\left(\inf A \cdot \arcsin \left(\frac{\inf A}{a}\right)+f(\inf A)^{\frac{1}{2}}\right)$.

(70) Suppose that $A \subseteq Z \subseteq]-1,1\left[\right.$ and $f=f_{1}-f_{2}$ and $f_{2}=\square^{2}$ and for every $x$ such that $x \in Z$ holds $f_{1}(x)=1$ and $f(x)>0$ and $x \neq 0$ and $\operatorname{dom}($ the function $\arccos )=Z \subseteq \operatorname{dom}\left(\operatorname{id}_{Z}\right.$ (the function $\left.\left.\arccos \right)-\left(\square \frac{1}{2}\right) \cdot f\right)$. Then $\int_{A}($ the function $\arccos )(x) d x=\sup A \cdot \arccos \sup A-f(\sup A)^{\frac{1}{2}}-$ $\left(\inf A \cdot \operatorname{arccosinf} A-f(\inf A)^{\frac{1}{2}}\right)$.

(71) Suppose that $A \subseteq Z \subseteq]-1,1\left[\right.$ and $f=f_{1}-f_{2}$ and $f_{2}=\square^{2}$ and for every $x$ such that $x \in Z$ holds $f_{1}(x)=a^{2}$ and $f(x)>0$ and $f_{3}(x)=\frac{x}{a}$ and $-1<f_{3}(x)<1$ and $x \neq 0$ and $a>0$ and $\operatorname{dom}\left((\right.$ the function $\left.\arccos ) \cdot f_{3}\right)=$ $Z=\operatorname{dom}\left(\operatorname{id}_{Z}\left((\right.\right.$ the function $\left.\left.\arccos ) \cdot f_{3}\right)-\left(\square^{\frac{1}{2}}\right) \cdot f\right)$ and ((the function $\left.\arccos ) \cdot f_{3}\right)\left\lceil A\right.$ is continuous. Then $\int_{A}\left((\right.$ the function $\left.\arccos ) \cdot f_{3}\right)(x) d x=$ $\sup A \cdot \arccos \left(\frac{\sup A}{a}\right)-f(\sup A)^{\frac{1}{2}}-\left(\inf A \cdot \arccos \left(\frac{\inf A}{a}\right)-f(\inf A)^{\frac{1}{2}}\right)$.

(72) Suppose that

(i) $A \subseteq Z$,

(ii) $Z \subseteq]-1,1[$,

(iii) $f_{2}=\square^{2}$, 
(iv) for every $x$ such that $x \in Z$ holds $f_{1}(x)=1$,

(v) $Z=\operatorname{dom}$ (the function arctan), and

(vi) $Z=\operatorname{dom}\left(\operatorname{id}_{Z}\right.$ the function $\arctan -\frac{1}{2}\left((\right.$ the function $\left.\ln ) \cdot\left(f_{1}+f_{2}\right)\right)$ ).

Then $\int_{A}($ the function $\arctan )(x) d x=\sup A \cdot \arctan \sup A-\frac{1}{2} \cdot \ln (1+$ $\left.(\sup A)^{2}\right)-\left(\inf A \cdot \arctan \inf A-\frac{1}{2} \cdot \ln \left(1+(\inf A)^{2}\right)\right)$.

(73) Suppose that

(i) $A \subseteq Z$,

(ii) $Z \subseteq]-1,1[$,

(iii) $f_{2}=\square^{2}$,

(iv) for every $x$ such that $x \in Z$ holds $f_{1}(x)=1$,

(v) $\operatorname{dom}$ (the function arccot) $=Z$, and

(vi) $Z=\operatorname{dom}\left(\operatorname{id}_{Z}\right.$ the function $\operatorname{arccot}+\frac{1}{2}\left((\right.$ the function $\left.\ln ) \cdot\left(f_{1}+f_{2}\right)\right)$ ).

Then $\int_{A}($ the function $\operatorname{arccot})(x) d x=\left(\sup A \cdot \operatorname{arccot} \sup A+\frac{1}{2} \cdot \ln (1+\right.$ $\left.\left.(\sup A)^{2}\right)\right)-\left(\inf A \cdot \operatorname{arccotinf} A+\frac{1}{2} \cdot \ln \left(1+(\inf A)^{2}\right)\right)$.

\section{REFERENCES}

[1] Czesław Byliński. Partial functions. Formalized Mathematics, 1(2):357-367, 1990.

[2] Czesław Byliński and Piotr Rudnicki. Bounding boxes for compact sets in $\mathcal{E}^{2}$. Formalized Mathematics, 6(3):427-440, 1997.

[3] Chuanzhang Chen. Mathematical Analysis. Higher Education Press, Beijing, 1978.

[4] Noboru Endou and Artur Korniłowicz. The definition of the Riemann definite integral and some related lemmas. Formalized Mathematics, 8(1):93-102, 1999.

[5] Noboru Endou, Katsumi Wasaki, and Yasunari Shidama. Definition of integrability for partial functions from $\mathbb{R}$ to $\mathbb{R}$ and integrability for continuous functions. Formalized Mathematics, 9(2):281-284, 2001.

[6] Artur Korniłowicz and Yasunari Shidama. Inverse trigonometric functions arcsin and arccos. Formalized Mathematics, 13(1):73-79, 2005.

[7] Jarosław Kotowicz. Convergent sequences and the limit of sequences. Formalized Mathematics, 1(2):273-275, 1990.

[8] Jarosław Kotowicz. Partial functions from a domain to a domain. Formalized Mathematics, 1(4):697-702, 1990.

[9] Jarosław Kotowicz. Partial functions from a domain to the set of real numbers. Formalized Mathematics, 1(4):703-709, 1990.

[10] Jarosław Kotowicz. Real sequences and basic operations on them. Formalized Mathematics, 1(2):269-272, 1990.

[11] Xiquan Liang and Bing Xie. Inverse trigonometric functions arctan and arccot. Formalized Mathematics, 16(2):147-158, 2008, doi:10.2478/v10037-008-0021-3.

[12] Takashi Mitsuishi and Yuguang Yang. Properties of the trigonometric function. Formalized Mathematics, 8(1):103-106, 1999.

[13] Konrad Raczkowski. Integer and rational exponents. Formalized Mathematics, 2(1):125130, 1991.

[14] Konrad Raczkowski and Paweł Sadowski. Real function differentiability. Formalized Mathematics, 1(4):797-801, 1990.

[15] Konrad Raczkowski and Paweł Sadowski. Topological properties of subsets in real numbers. Formalized Mathematics, 1(4):777-780, 1990.

[16] Yasunari Shidama. The Taylor expansions. Formalized Mathematics, 12(2):195-200, 2004. 
[17] Andrzej Trybulec and Czesław Byliński. Some properties of real numbers. Formalized Mathematics, 1(3):445-449, 1990.

[18] Andrzej Trybulec and Yatsuka Nakamura. On the decomposition of a simple closed curve into two arcs. Formalized Mathematics, 10(3):163-167, 2002.

[19] Zinaida Trybulec. Properties of subsets. Formalized Mathematics, 1(1):67-71, 1990.

[20] Peng Wang and Bo Li. Several differentiation formulas of special functions. Part V. Formalized Mathematics, 15(3):73-79, 2007, doi:10.2478/v10037-007-0009-4.

[21] Edmund Woronowicz. Relations and their basic properties. Formalized Mathematics, 1(1):73-83, 1990.

[22] Edmund Woronowicz. Relations defined on sets. Formalized Mathematics, 1(1):181-186, 1990.

[23] Yuguang Yang and Yasunari Shidama. Trigonometric functions and existence of circle ratio. Formalized Mathematics, 7(2):255-263, 1998.

Received October 14, 2008 